\title{
Clinical Outcomes in Multiple Sclerosis Patients Following Venous Angioplasty
}

Can J Neurol Sci. 2013; 40: 141-143

In this issue of the Canadian Journal of Neurological Sciences, Pryse-Phillips et $\mathrm{al}^{1}$ report the results of a one-year observational study of patients with multiple sclerosis (MS) who underwent venous angioplasty for chronic cerebrospinal venous insufficiency (CCSVI), which has been proposed to be the underlying cause of MS. ${ }^{2}$ Subjects who underwent the procedure $(n=30)$ demonstrated a significant improvement in subjective symptomatology one month post-procedure, as measured by the multiple sclerosis impact scale (MSIS-29), which is a patientreported outcome scale. However, over the follow-up period of one year, the magnitude of the perceived benefit decreased by $15 \%$. In contrast, objective measures of neurological function, including the expanded disability status scale (EDSS) and the multiple sclerosis functional composite (MSFC) scores showed no evidence of improvement following venoplasty. Interestingly, control subjects with MS who had not undergone venoplasty actually showed an improvement in EDSS at the one-year follow-up point. Finally, there were no differences in subjective (MSIS-29) or objective (EDSS, MSFC) clinical measures in subjects with CT-venogram (CTV) evidence of restenosis at one year post-venoplasty, vs. those without re-stenosis.

Although a number of significant limitations prevent the formulation of any definitive conclusions from this study, it is nonetheless an important contribution to the growing body of CCSVI literature. Pryse-Phillips et $\mathrm{al}^{1}$ report longitudinal clinical follow-up on a "real-world" sample of MS patients postvenoplasty, performed by a neurologist blinded to the patient's clinical status. Most noteworthy from this study is the fact that subjective patient-reported clinical benefits were not corroborated by objective measures of clinical function. This observation does not support the use venoplasty in the treatment of MS. Furthermore, the lack of a measurable clinical difference when comparing subjects who experienced re-stenosis vs. those that did not post-venoplasty sheds further doubt on the efficacy of venoplasty in MS, and on the causal role of CCSVI in MS.

Significant limitations in this study include the small sample size and the observational study design, which likely resulted in confounding variables that were not accounted for in the analysis. In addition, although there was a control group of MS patients that did not undergo venoplasty $(n=10)$, these patients did not undergo serial imaging or clinical examinations other than the EDSS. Thus, direct comparisons of all outcomes between the control and venoplasty groups were not possible. The authors suggest that the lack of change in MRI measures in the venoplasty group over the follow-up period supports the lack of a benefit of CCSVI, however, this conclusion is not valid without a control group comparison. Finally, the validity of comparing clinical measures over the one-year follow-up period in subjects with re-stenosis on follow-up CTV (performed at one year post-venoplasty) vs. subjects without re-stenosis is unclear, since the exact time point when subjects underwent re-stenosis is impossible to ascertain based on these data. Notwithstanding these limitations, however, this study provides important clinical data on this topic, and helps fill an important gap in the literature pertaining to patients treated with venoplasty for CCSVI.

Since the first report of CCSVI by Zamboni et al in 2009, 3,4 CCSVI has been an intensely debated topic in MS. Media sensationalization of CCSVI has culminated in patients independently seeking out venoplasty, an invasive procedure with significant risks, ${ }^{5}$ despite the lack of definitive evidence in support of the efficacy of this procedure.

A number of independent groups have assessed the prevalence of CCSVI in MS patients, and have found no evidence of increased CCSVI in MS patients in comparison to healthy controls or subjects with other neurological disorders, thus failing to replicate Zamboni's findings. ${ }^{6-13}$ Although some groups have demonstrated an increase in CCSVI in MS, they were unable to demonstrate the presence of CCSVI to the same extent as Zamboni's original study, ${ }^{4}$ which reported a prevalence of $100 \% .^{14,15}$

A recent meta-analysis by Laupacis et al found a positive association between CCSVI and MS; however, the conclusion from this study was that the meta-analysis results must be interpreted with caution since there was significant heterogeneity amongst included studies, and all included studies were either unblinded, or did not report on the success of blinding. ${ }^{16}$ Blinding is essential to prevent ascertainment bias in the assessment of CCSVI which is typically ascertained by ultrasonography, an exquisitely operator-dependent imaging technique.

Since this meta-analysis, ${ }^{16}$ a number of additional studies have been reported that cast further doubt on the causative role of CCSVI in MS. Comi et al reported results from the "CosMO study", which is the largest and most rigorously conducted CCSVI study to date. ${ }^{13}$ CosMO was a multicenter trial that recruited MS patients $(n=1871)$ from 35 different centers. Ultrasonography was performed by trained technicians according to a pre-defined protocol, and analysis of ultrasounds took place at a central facility by an expert panel of blinded investigators. The CosMO study found that the prevalence of CCSVI was not different between MS patients, healthy controls, or subjects with other neurological disorders. The scale of this study, coupled with the sound methodology provides strong evidence against a causative role for CCSVI in MS.

Another recent methodologically sound study by Barreto et al found no difference in CCSVI prevalence in MS subjects $(n=198)$ vs. those with other neurological disease or healthy controls. ${ }^{17}$ Three recent studies found no differences in CCSVI prevalence in pediatric MS patients vs. healthy controls. Zivadinov et al conclude that these findings point against CCSVI having a primary causative role in MS. ${ }^{18-20}$ 
Clinical outcomes in MS patients post-venoplasty have been reported in only a handful of studies.

Zamboni et al reported improvement in a variety of subjective, objective, and imaging-based measures in MS patients $(n=65)$ after a median follow-up of 18 months. ${ }^{3}$ The open-label extension of this study after a median follow-up of two years $(n=29)$ showed clinical benefit (improved EDSS, lower relapse rate).$^{21}$ However, a major limitation of this study was the open-label design, making it necessary to interpret both objective and subjective clinical outcomes with caution.

Hubbard et al followed MS patients $(n=259)$ at one and six months post-venoplasty, and found a sustained benefit at six months in the MSIS-29, a subjective outcome measure. ${ }^{22}$

A study by Kostecki et al demonstrated improvement in subjective clinical measures (heat intolerance scale, fatigue severity scale, MSIS-29) at three-months post-venoplasty, but there was no sustained improvement of MSIS-29 at six months. Importantly, there was no demonstrable benefit in any objective clinical measures. In addition, there were no observable clinical differences in subjects who had experienced re-stenosis vs. those that did not. ${ }^{23}$ More recently, Ghezzi et al reported the results of an observational study in a large sample of MS patients $(n=462)$ who had undergone venoplasty. Although $53 \%$ of subjects reported subjective benefit, there was no evidence of any change in EDSS, an objective clinical measure. ${ }^{24}$

Taken together, the existing clinical follow-up studies of MS patients post-venoplasty conducted by independent groups suggest that the observed clinical benefit, if any, is in subjective measures alone, which can be heavily influenced by patient expectations. The lack of a concomitant improvement in objective clinical measures undermines the view that this procedure has clinical benefit. Observations from Pryse-Phillips et $\mathrm{al}^{1}$ cast further doubt on the clinical efficacy of venoplasty in MS patients, and have the advantage over other existing studies of having utilized blinded neurological assessments.

Scientific discovery requires a fine balance between openness to new ideas and their rigorous scrutiny. With the CCSVI hypothesis, the pendulum appears to have swung too quickly and too far to one side, which has resulted in a large number of patients seeking out a potentially risky procedure that has yet to demonstrate strong evidence of clinical efficacy. Although future studies will allow for a more definitive verdict on the role of CCSVI in MS, based on the existing evidence, the likelihood that CCSVI plays a substantial role in MS pathogenesis seems highly improbable. As such, clinicians should strongly discourage the pursuit of venoplasty in patients with MS, as this is not a benign procedure, with reported serious adverse events. ${ }^{5}$

Although there is accumulating evidence against CCSVI's role in causing or even being associated with MS, planned or ongoing interventional trials include the Evaluation of Angioplasty in the Treatment of CCSVI in Multiple Sclerosis trial (Siskin et al), ${ }^{25}$ the BRAVE DREAMS trial (Zamboni et al), ${ }^{26}$ and a Canadian clinical trial that is a collaborative initiative between the federal government, three provinces (British Columbia, Manitoba, and Quebec), and the MS Society of Canada. ${ }^{27}$ Furthermore, Brad Wall, the premier of Saskatchewan, has announced \$2.2 million to fund MS patients in Saskatchewan to support enrollment in the CCSVI trial by Siskin et al. ${ }^{28}$

In a recent commentary, Zivadinov et al appropriately state that "future studies aimed to better understand the associated effects of CCSVI and MS, as well as their effects on general health, should be further explored before larger interventional studies are undertaken." 29 Given the overall evidence to date that suggests that CCSVI is unusual in MS, and present with equal prevalence in MS patients vs. control subjects, the question of the ethical justification for doing such interventional clinical trials has become even more pressing.

\author{
Jiwon $\mathrm{Oh}^{1,2}$, Paul O'Connor ${ }^{2}$ \\ ${ }^{1}$ Johns Hopkins University, Baltimore, Maryland, United States \\ ${ }^{2}$ University of Toronto, Toronto, Ontario, Canada
}

\section{REFERENCES}

1. Pryse-Phillips W, Stefanelli M, Murphy-Peddle K, Barrett B. An observational study of venoplasty in patients with multiple sclerosis patients. Can J Neurol Sci. 2013;40(2):203-9.

2. Zamboni P, Galeotti R, Menegatti E, et al. Chronic cerebrospinal venous insufficiency in patients with multiple sclerosis. J Neurol Neurosurg Psychiatry. 2009;80:392-9.

3. Zamboni P, Galeotti R, Menegatti E, et al. A prospective open-label study of endovascular treatment of chronic cerebrospinal venous insufficiency. J Vasc Surg. 2009;50:1348-58.e1-3.

4. Zamboni P, Menegatti E, Galeotti R, et al. The value of cerebral Doppler venous haemodynamics in the assessment of multiple sclerosis. J Neurol Sci. 2009;282:21-7.

5. Burton JM, Alikhani K, Goyal M, et al. Complications in MS patients after CCSVI procedures abroad (Calgary, AB). Can J Neurol Sci. 2011;38:741-6.

6. Zivadinov RMK, Valnarov V, Kilanowski C, et al. Prevalence, sensitivity and specificity of chronic cerebrospinal venous insufficiency in other neurologic diseases. A case-control study. In: European Congress for Treatment and Research in Multiple Sclerosis (ECTRIMS). Lyon, France: 2012.

7. Doepp F, Paul F, Valdueza JM, Schmierer K, Schreiber SJ. No cerebrocervical venous congestion in patients with multiple sclerosis. Ann Neurol. 2010;68:173-83.

8. Mayer CA, Pfeilschifter W, Lorenz MW, et al. The perfect crime? CCSVI not leaving a trace in MS. J Neurol Neurosurg Psychiatry. 2011;82:436-40.

9. Auriel E, Karni A, Bornstein NM, Nissel T, Gadoth A, Hallevi H. Extra-cranial venous flow in patients with multiple sclerosis. JNeurol Sci. 2011;309:102-4.

10. Wattjes MP, van Oosten BW, de Graaf WL, et al. No association of abnormal cranial venous drainage with multiple sclerosis: a magnetic resonance venography and flow-quantification study. $\mathrm{J}$ Neurol Neurosurg Psychiatry. 2011;82:429-35.

11. Blinkenberg M, Akeson P, Sillesen H, et al. Chronic cerebrospinal venous insufficiency and venous stenoses in multiple sclerosis. Acta Neurol Scand. 2012;126:421-7.

12. Baracchini C, Perini P, Calabrese M, Causin F, Rinaldi F, Gallo P. No evidence of chronic cerebrospinal venous insufficiency at multiple sclerosis onset. Ann Neurol. 2011;69:90-9.

13. Comi GB, Bertolotto A, Del Sette M, et al on behalf of the CoSMo study group. Chronic Cerebro-Spinal Venous Insufficiency (CCSVI) and global venous haemodynamics in multiple sclerosis: the CoSMo study. In: European Congress for Treatment and Research in Multiple Sclerosis (ECTRIMS). Lyon, France: 2012.

14. Patti F, Nicoletti A, Leone C, et al. Multiple sclerosis and CCSVI: a population-based case control study. PloS one. 2012;7:e41227.

15. Zivadinov R, Marr K, Cutter G, et al. Prevalence, sensitivity, and specificity of chronic cerebrospinal venous insufficiency in MS. Neurol. 2011;77:138-44. 
16. Laupacis A, Lillie E, Dueck A, et al. Association between chronic cerebrospinal venous insufficiency and multiple sclerosis: a meta-analysis. CMAJ. 2011;183:e1203-12.

17. Barreto AB, Bui T, Jamelka J, et al. A study of CCSVI with imaging-blinded assessment: Neurosonography update. In: European Congress for Treatment and Research in Multiple Sclerosis (ECTRIMS). Lyon, France: 2012.

18. Amato M, Saia V, Hakiki B, et al. No association between chronic cerebrospinal venous insufficiency and pediatric-onset multiple sclerosis (published online ahead of print 2012 Apr 18). Multiple sclerosis. [cited 2012 Nov 12] Available from:http://msj. sagepub.com/content/early/2012/04/17/1352458512445943.long.

19. Banwell BL, Traubici J, Khan S, et al. No evidence for impairment in venous haemodynamics in children or young adults with paediatric-onset multiple sclerosis. In: European Congress for Treatment and Research in Multiple Sclerosis (ECTRIMS). Lyon, France: 2012.

20. Zivadinov RY, Marr K, Valnarov V, et al. Prevalence, sensitivity and specificity of chronic cerebrospinal venous insufficiency in paediatric multiple sclerosis. In: European Congress for Treatment and Research in Multiple Sclerosis (ECTRIMS). Lyon, France: 2012.

21. Salvi F, Bartolomei I, Buccellato E, Galeotti R, Zamboni P. Venous angioplasty in multiple sclerosis: neurological outcome at two years in a cohort of relapsing-remitting patients. Funct Neurol. 2012;27:55-9.

22. Hubbard D, Ponec D, Gooding J, Saxon R, Sauder H, Haacke M. Clinical improvement after extracranial venoplasty in multiple sclerosis. J Vasc Interv Radiol. 2012;23:1302-8.
23. Kostecki J, Zaniewski M, Ziaja K, et al. An endovascular treatment of Chronic Cerebro-Spinal Venous Insufficiency in multiple sclerosis patients - 6 month follow-up results. Neuro Endocrinol Lett. 2011;32:557-62.

24. Ghezzi AA, Amato MP, Balgera R, et al and MS Study Group, Italian Society of Neurology. Endovascular treatment of CCSVI in patients with multiple sclerosis: clinical outcome of an Italian cohort of 462 cases. In: European Congress for Treatment and Research in Multiple Sclerosis (ECTRIMS). Lyon, France: 2012.

25. Evaluation of angioplasty in the treatment of chronic cerebrospinal venous insufficiency (CCSVI) in multiple sclerosis [online]. [updated 2010 Sept 20; cited 2012 Nov 17]. Available at: http://clinicaltrials.gov/ct2/show/NCT01201707?term=ccsvi\&ra nk=2.

26. Zamboni P, Bertolotto A, Boldrini P, et al. Efficacy and safety of venous angioplasty of the extracranial veins for multiple sclerosis. Brave Dreams Study (Brain Venous Drainage Exploited Against Multiple Sclerosis): study protocol for a randomized controlled trial. Trials. 2012;13:183.

27. MS Society partners in CCSVI clinical trial [online]. [updated 2012 Sept 28; cited 2012 Nov 12]. Available at: http://mssociety.ca/ en/releases/nr 20120928.htm.

28. MS clinical trial applications open to Saskatchewan patients [online]. updated 2012 Jan 12; cited 2012 Nov 12]. Available at: http://www.gov.sk.ca/news?newsId=220326aa-d59e-4f60-8d8ff6b2da096ade.

29. Zivadinov R, Weinstock-Guttman B. Role of venoplasty for treatment of multiple sclerosis: value of open-label studies and surrogate treatment outcomes. J Vasc Interv Radiol. 2012;23: 1308-10. 